\title{
Editorial
}

\section{Applications of Fuzzy Ensemble Approaches in Modeling, Forecasting, and Control}

\author{
Toly Chen, ${ }^{1}$ Katsuhiro Honda, ${ }^{2}$ Pedro Ponce, ${ }^{3}$ T. Warren Liao, ${ }^{4}$ and Yi-Chi Wang ${ }^{1}$ \\ ${ }^{1}$ Department of Industrial Engineering and Systems Management, Feng Chia University, Taichung 407, Taiwan \\ ${ }^{2}$ Graduate School of Engineering, Osaka Prefecture University, Osaka 599-8531, Japan \\ ${ }^{3}$ Posgrados en Ciencias de la Ingeniería, TEC de Monterrey, Campus Ciudad de México, Tlalpan C.P. 14380, Mexico \\ ${ }^{4}$ Department of Mechanical and Industrial Engineering, Louisiana State University, Baton Rouge, LA 70803, USA
}

Correspondence should be addressed to Toly Chen; tcchen@fcu.edu.tw

Received 20 November 2013; Accepted 20 November 2013

Copyright (C) 2013 Toly Chen et al. This is an open access article distributed under the Creative Commons Attribution License, which permits unrestricted use, distribution, and reproduction in any medium, provided the original work is properly cited.

Fuzzy systems have been successfully applied to various fields. However, most applications are based on a single fuzzy system. The simultaneous use of multiple fuzzy systems may be able to achieve better performances. These systems, homogeneous or heterogeneous, collaborate to enhance the effectiveness or efficiency of solving a problem. Based on this point of view, the ensemble of fuzzy systems has been widely used for the modeling, forecasting, and control of complex systems under an uncertain, restricted, or subjective environment. The purpose of this special issue is to provide the details of recent advances in developing fuzzy ensemble approaches and their applications. The target audiences are researchers in engineering mathematics, information engineering, information management, artificial intelligence, and computational intelligence, as well as practicing managers/engineers. After a very strict review process, only five papers from researchers around the world were finally accepted. A brief summary of each is described below.

M.-Y. Shan et al. proposed a combination of cloud model and quantum-behaved particle swarm optimization (COCQPSO) to solve the problems of fitting complex functions and clustering various datasets. They also investigated the relation between Gaussian and fat-tail distributions, so as to construct a fat-tail distribution from Gaussian distribution in an iterative way to depict more uncertain phenomena.

H.-C. Wu and T. Chen proposed a fuzzy-neural ensemble and geometric rule fusion approach to optimize the performance of job dispatching in a wafer fabrication factory. Two dispatching rules were fused, and job slacks were diversified by maximizing the geometric mean of the neighboring distances of slacks. In addition, the fuzzy c-means (FCM) and back propagation network (BPN) ensemble approach was also applied to estimate the remaining cycle time of a job, which then served as an input to the new rule.

The existing nonlinear programming (NLP) based fuzzy collaborative intelligence methods cannot distinguish between the different expert opinions. It is also not easy to find the global optimal solution using these methods. In order to solve these problems and to improve the performance of the semiconductor yield forecasting, T. Chen and Y.-C. Wang proposed a quadratic programming (QP) based fuzzy collaborative intelligence approach.

$\mathrm{H}$. Ponce et al. presented a new fuzzy inference model based on artificial hydrocarbon networks. In addition, the fuzzy-molecular inference model (FIM-model) was also proposed to partition the output space in the defuzzification step according to the information from the molecular units. Such molecules are linguistic units that can be partially understandable through the organized structure of the topology and the metadata parameters involved in the artificial hydrocarbon network.

$\mathrm{X}$. Liu and Y. Chen proposed a systematic approach to optimize the $h$ value in a fuzzy linear regression (FLR) problem, based on the minimum fuzziness criteria with symmetric triangular fuzzy numbers (TFNs). They defined a new concept of credibility to evaluate the performance of 
an FLR model with different $h$ values for a set of sample data pairs. Based on this concept, a mathematical programming model was formulated and solved to optimize the $h$ value.

\section{Acknowledgment}

We sincerely thank all the authors and reviewers for their valuable contributions to this special issue.

Toly Chen Katsuhiro Honda Pedro Ponce

T. Warren Liao Yi-Chi Wang 


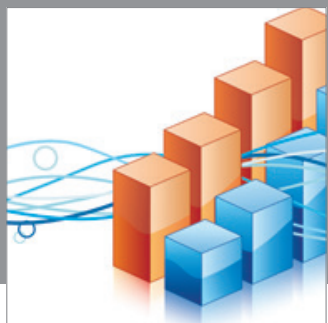

Advances in

Operations Research

mansans

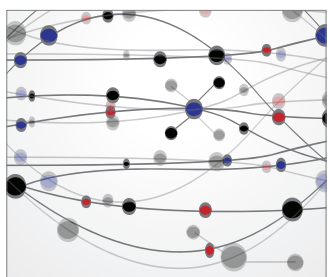

The Scientific World Journal
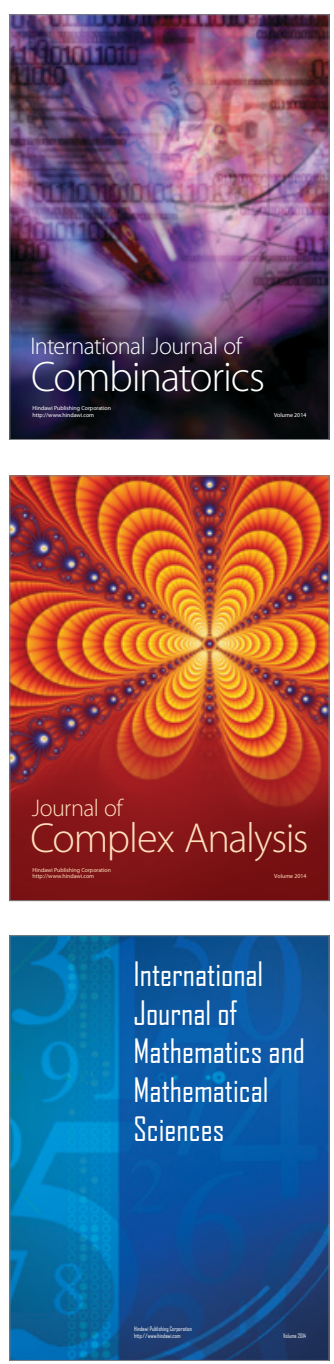
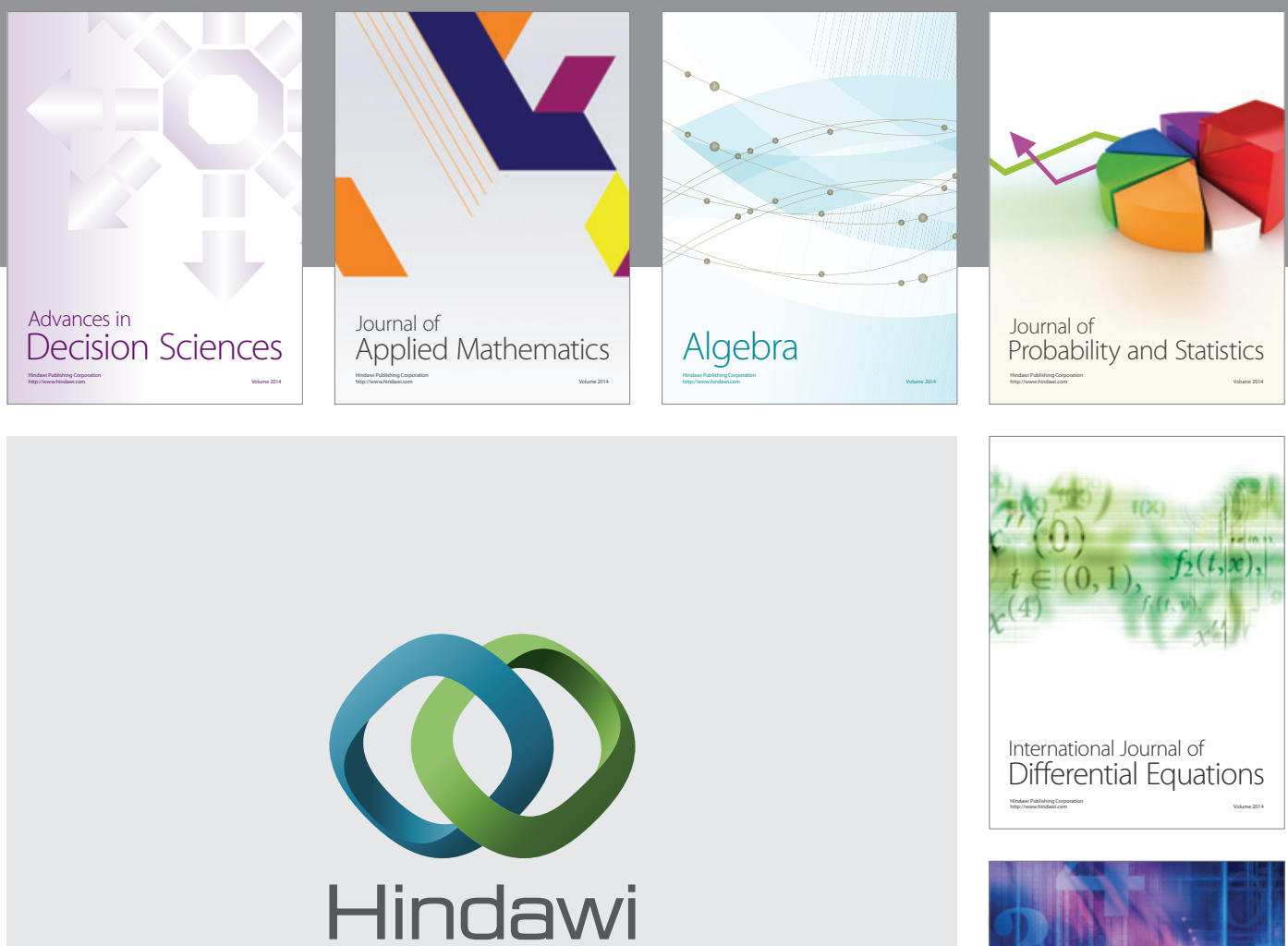

Submit your manuscripts at http://www.hindawi.com
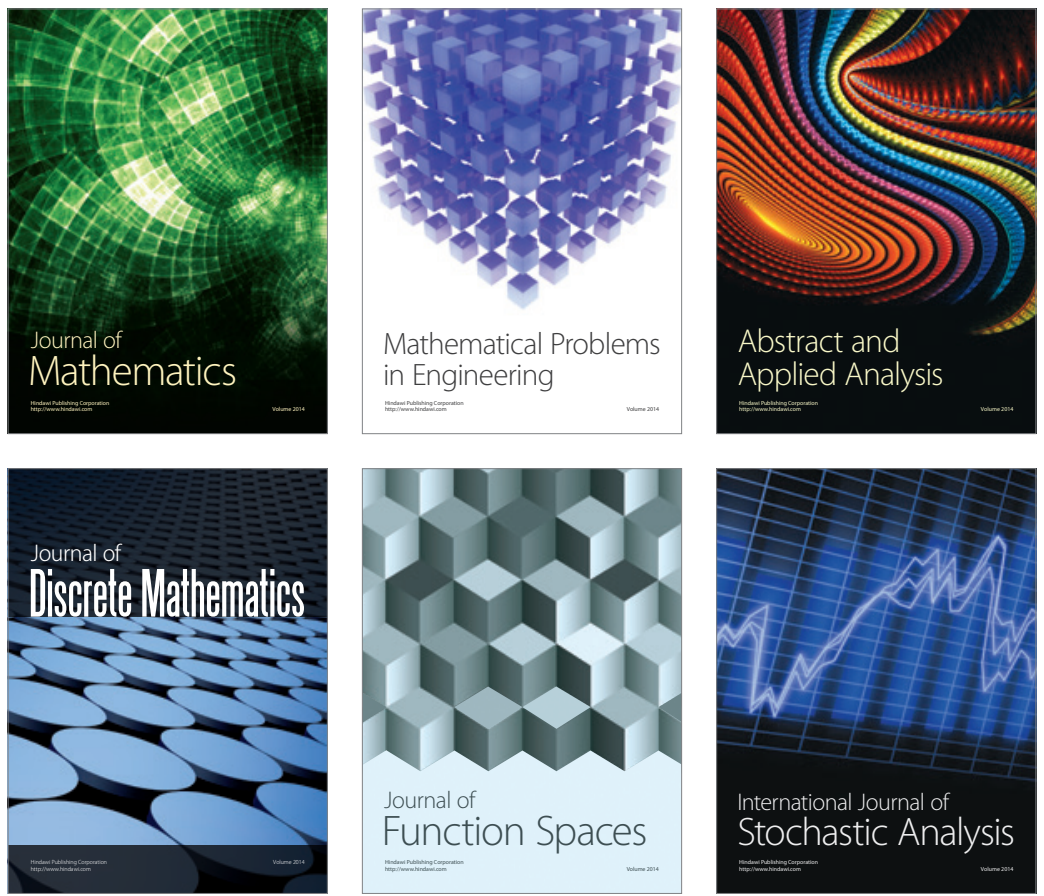

Journal of

Function Spaces

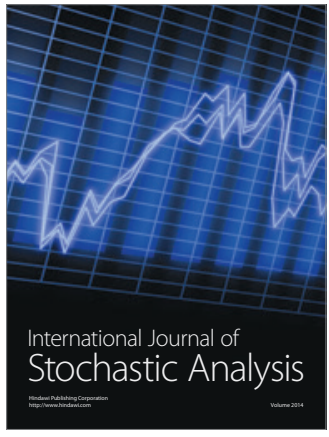

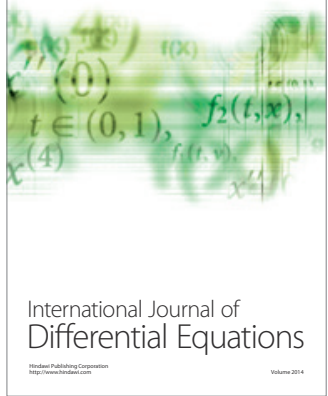
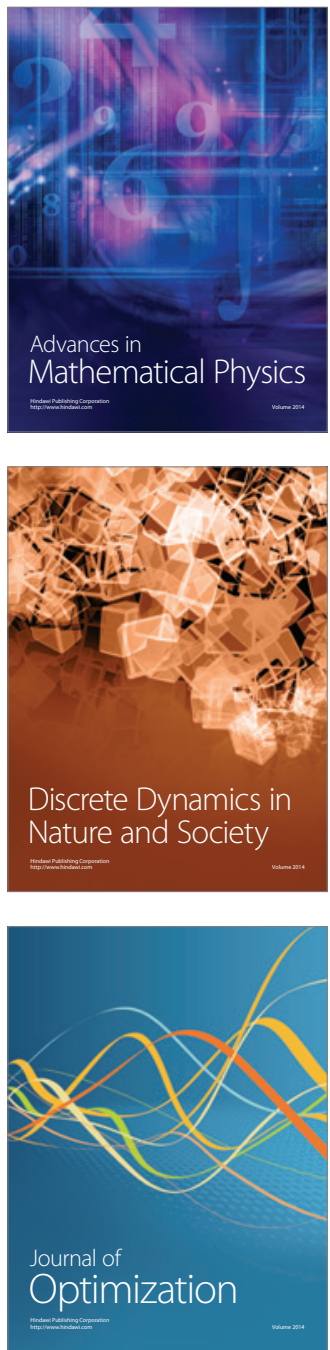